\title{
Pédagogie coopérative: pratiques déclarées et facteurs d'appropriation
}

Yann Volpé et Céline Buchs, Université de Genève

Cette étude documente l'appropriation de la pédagogie coopérative par les enseignant$e-s$ ayant suivi une initiation en formation continue. Ces enseignant-e-s rapportent mettre en place des travaux de groupes coopératifs sur 25\% du temps d'enseignement en moyenne et déclarent préparer les élèves à coopérer et organiser leurs interactions selon les principes de cette pédagogie. Cependant, le lien entre les variables précédentes est faible. Les répondants expriment de la difficulté à gérer le temps et à opérationnaliser l'interdépendance positive. Ils-elles identifient les objectifs d'apprentissage et les moyens d'enseignement comme un frein à leur pratique; la perception des bénéfices pour les élèves ainsi que le soutien collégial et institutionnel sont considérés comme des leviers.

Le Plan d'Études Romand (PER) en vigueur sur le Canton de Genève accorde une importance particulière à la collaboration entre les élèves (Conférence intercantonale de l'instruction publique, CIIP, 2010). Les élèves sont ainsi encouragés à apprendre ensemble. Dans ce contexte, il a été démontré que le travail entre pairs faciliterait l'apprentissage (Buchs, Lehraus \& Crahay, 2012) et stimulerait les activités intellectuelles tout en permettant des actes conscients et réfléchis (Peyrat-Malaterre, 2011). La pédagogie coopérative propose de préparer les élèves à coopérer et d'organiser le travail de manière à permettre aux élèves d'interagir de manière constructive. De nombreuses analyses ont souligné ses bénéfices sur les apprentissages scolaires, les relations sociales et la motivation (notamment Johnson \& Johnson, 2009).

Malgré ces bénéfices, c'est une pratique complexe et exigeante (Buchs, Filippou, Pulfrey \& Volpé, 2017; Kutnick, Ota \& Berdondini, 2008; Sharan, 2010) qui reste minoritaire dans les classes à Genève (Buchs et al., 2017). L'objectif de cette étude est de documenter ce qu'il en est pour des enseignante-s qui se sont montré-e-s intéressé-e-s par cette pédagogie en suivant volontairement une initiation à la pédagogie coopérative en formation continue. 


\section{La pédagogie coopérative}

Historiquement, la coopération en éducation s'est développée en parallèle sur deux continents: en Europe, sous l'influence de Freinet et de Cousinet, puis aujourd'hui à travers les travaux de Connac (2010) avec l'objectif de lutter contre l'école-caserne et de donner du sens aux savoirs au travers d'une communauté d'apprentissage ; en Amérique du Nord, dans les années septante, avec le courant Cooperative learning. Celui-ci regroupe différentes méthodes visant à structurer des travaux de groupes dans les classes pour une meilleure efficacité (Teamsachievement divisions, Team assisted individualization, Jigsaw, Learning together, Academic controversy, Complex instruction, Group structural approach; voir Abrami et al., 1996 pour une présentation en français ou Sharan, 1999, pour une présentation par les auteurs originaux). C'est dans cette deuxième perspective que nous nous inscrivons.

Nous différencions ainsi le travail de groupe de l'apprentissage coopératif. Le travail de groupe consiste à placer les élèves ensemble en leur demandant de travailler conjointement. Il est courant d'observer certaines dérives dans ce type de travail (voir Toczek, 2004) qui peut contribuer à favoriser les élèves déjà avantagés (Cohen, 2002). Le courant nord-américain de l'apprentissage coopératif place les interactions sociales entre pairs au service des apprentissages scolaires (Buchs, 2017; Topping, Buchs, Duran \& van Keer, 2017): les élèves se soutiennent, s'encouragent et valorisent les efforts et contributions de chacun; ils synthétisent et s'enseignent mutuellement; ils confrontent et coconstruisent les nouveaux savoirs. Toutes les méthodes d'apprentissage coopératif proposent des principes communs pour organiser ces interactions constructives lors des travaux structurés (Davidson \& Major, 2014; Topping et al., 2017). La méthode Learning together proposée par le «Cooperative learning center» de Minneapolis (p. ex. Johnson, Johnson \& Holubec, 2008) et particulièrement diffusée en francophonie (voir p. ex. Howden \& Kopiec, 2000; Johnson \& Johnson, 1998; Rouiller \& Howden 2010) ajoutent des principes supplémentaires (Buchs, 2017). Dans la lignée de Topping et al. (2017), nous proposons de distinguer les principes de l'apprentissage coopératif qui organisent les interactions entre pairs lors des travaux de groupes (coopérer pour apprendre) et les principes supplémentaires pour préparer les apprenants à coopérer (apprendre à coopérer) et parlons de pédagogie coopérative pour désigner les deux ensembles de principes (dès à présent PC). Cette appellation a l'avantage de souligner le rôle primordial de l'enseignant-e.

\section{Organiser les interactions lors du travail}

Trois principes sont essentiels (Davidson \& Major, 2014; Topping et al., 2017): 1) L'interdépendance positive constitue la véritable clé de voûte. Elle existe dès le moment où un élève perçoit qu'il peut atteindre son but lorsque les autres membres de son groupe atteignent également le leur (Johnson et al., 2008). 
Les élèves sont donc complémentaires et les efforts de tous sont nécessaires. L'enseignant-e doit ainsi assurer l'interdépendance positive liée aux buts (suffisante et indispensable, Johnson \& Johnson, 1989) et peut ajouter d'autres interdépendances en fonction des activités (Jonson et al., 2008).

2) La responsabilité individuelle permet à chaque élève de se sentir responsable de sa contribution au travail de groupe et de sa progression dans les apprentissages. Chaque élève encourage ses pairs à adopter une attitude identique.

3) Une tâche de groupe ne peut être réalisée sans l'apport de tous les membres. Cette tâche est effectuée dans des groupes restreints de deux à cinq élèves pour permettre les interactions individualisées.

\section{Préparer les apprenants à coopérer}

Malgré les trois principes précédents, les élèves ne coopèrent pas de manière spontanée (Buchs \& Butera, 2015). Pour qu'ils acquièrent les compétences sociales et cognitives, une préparation à la coopération est nécessaire. Elle inclut trois principes (Buchs, 2017; Johnson et al., 2008; Topping et al., 2017):

1) L'établissement d'un climat de classe propice aux apprentissages qui s'opérationnalise par des relations de confiance entre les différents acteurs, une motivation orientée vers la maitrise et des relations sociales soutenantes (Buchs, 2017). L'enseignant-e introduit des activités permettant aux élèves de se sentir acceptés dans le groupe et d'oser travailler ensemble.

2) L'enseignement explicite des habiletés coopératives permet un travail efficace (Johnson et al., 2008). L'enseignant-e pointe les habiletés nécessaires et chacune d'elles fait l'objet d'un travail spécifique sur la manière dont celle-ci peut se concrétiser en gestes et en paroles. L'enjeu ici est de travailler explicitement avec les élèves les compétences sociales nécessaires à guider leur activité de manière à ce que tous aient accès aux conditions d'apprentissage et de réussite.

3) La réflexion critique sur les processus de groupe est instaurée après tout travail coopératif. L'enseignant-e propose aux élèves de réfléchir sur le déroulement de l'activité et sur la manière d'améliorer leur travail lors de la prochaine séance (Johnson et al., 2008).

\section{La PC dans les classes de L'enseignement primaire}

La mise en pratique de la PC ne semble pas si évidente pour les enseignant-e-s (Gillies \& Boyle, 2010; Kutnick et al., 2008; Sharan, 2010) et cette pédagogie reste minoritaire parmi les pratiques professionnelles (Abrami, Poulsen \& Chambers, 2004; Buchs et al., 2017). Les observations en classe révèlent que les élèves travaillent collectivement ou individuellement la plus grande partie du temps (Pianta, Belsky, Houts, Morrison, 2007), ou sont assis en groupe sans travailler ensemble (Kutnick et al., 2008). Ce constat se retrouve dans le contexte genevois: plus de $40 \%$ des enseignant-e-s primaires rapportent pratiquer rarement ou occasionnellement des travaux de groupes coopératifs et seuls 33\% 
les utilisent régulièrement (Buchs et al., 2017). La présente étude a pour objectif de documenter dans quelle mesure les enseignant-e-s qui, dans leur parcours, ont manifesté un intérêt pour la PC, déclarent mettre en place le travail de groupe coopératif.

En plus du peu de temps dédié au travail entre pairs, Antil, Jenkins, Wayne et Vadasy (1998) soulignent un décalage entre les principes théoriques et les pratiques professionnelles: lorsqu'il s'agit de structurer les interactions, les enseignants ne mobilisent pas toujours les différents principes qu'ils perçoivent comme complexes à comprendre. Ainsi, les enseignant-e-s adaptent les propositions théoriques (Koutselini, 2008; Lehraus, 2002; Sabourin \& Lehraus, 2008), ce qui peut rendre la mise en œuvre peu adéquate et réduire les bénéfices (Lehraus, 2002). La présente étude vise également à documenter les principes que les enseignant-e-s déclarent introduire lorsqu'ils-elles proposent des travaux de groupes coopératifs.

\section{Des défis à surmonter pour mettre en pratique la pédagogie coopérative}

\section{Les difficultés potentielles}

Même lorsque les enseignant-e-s souhaitent mettre en pratique la $\mathrm{PC}$, la mise en œuvre reste délicate (Buchs et al., 2017; Gillies \& Boyle, 2010; Sharan, 2010). L'objectif de cette étude est d'identifier les difficultés exprimées par les enseignant-e-s qui ont choisi en formation continue de suivre une initiation à la PC. Nous pouvons donc supposer qu'ils-elles ont une perception positive de la coopération en classe et que l'initiation de deux jours leur permet d'acquérir certaines connaissances sur la PC. Il est donc intéressant de documenter les difficultés que ces enseignant-e-s identifient lors de la mise en place des travaux coopératifs.

\section{Difficulté à mettre en ouvre les différents principes}

Une première difficulté expérimentée peut résider dans le décalage entre les principes théoriques et les pratiques professionnelles documenté par Antil et al. (1998). Les enseignant-e-s, bien que sensibilisé-e-s à l'importance des interactions constructives, à la préparation à la coopération et à l'organisation des interactions, identifient les différents principes comme particulièrement complexes.

\section{Difficulté à modifier le lieu d'autorité}

La PC propose de transférer une partie de la responsabilité de l'enseignant-e aux élèves (Brody, 1998; Cohen, 1994; Lotan, 2004). Ce changement de lieu d'autorité est illustré par le fait qu' "un dispositif se substitue à l'enseignant-e [... réalisant] le double avantage d'un retrait de l'enseignant-e [...] et du maintien d'un cadre qui permet d'orienter et aider les élèves à [...] apprendre» (Foray, 2016, p.152). Cependant, certain-e-s enseignant-e-s craignent de ne plus contrôler ce 
qui se passe dans la classe (Baines, Rubie-Davies \& Blatchford, 2009; Cohen, 1994; Gillies \& Boyle, 2010; Koutselini, 2008) et redoutent les problèmes de discipline (Baines, Blatchford \& Kutnick, 2003; Blatchford, Kutnick, Baines \& Galton, 2003).

\section{Difficulté à endosser un nouveau rôle de régulateur-trice}

La PC représente un changement de perspective: l'enseignant-e est davantage centré-e sur le traitement de l'information, les stratégies cognitives et métacognitives, les méthodes de travail ainsi que les attitudes (Sabourin \& Lehraus, 2008). Il-elle devient observateur-trice et peut alors intervenir pour réguler les comportements et les activités cognitives (Brody, 1998; Cohen, 2004). Cette modification du rôle professionnel peut questionner l'enseignant-e (Abrami et al., 2004) qui n'incarne plus la posture traditionnelle de transmission des savoirs.

\section{Difficulté à travailler avec des groupes}

Gillies et Boyle (2010) mettent en avant les difficultés rencontrées lors de la composition des groupes. En effet, cette constitution implique un certain nombre de décisions (Topping et al., 2017): qui fait les groupes? sur quels critères? combien d'élèves? Une fois les groupes constitués, l'enseignant-e doit aussi arriver à composer avec les différences de rythme lors de l'avancement de la tâche.

\section{Difficulté à évaluer les élèves dans le travail de groupe}

Plusieurs recherches soulèvent le questionnement sur l'évaluation des travaux de groupes (Blatchford et al., 2003). Ces travaux soulignent la difficulté d'évaluer individuellement les membres d'un groupe qui produit des connaissances communes (Buchs et al., 2017; Cohen, 1994) et de prendre en compte simultanément des objectifs scolaires et coopératifs.

\section{Difficultés à créer des activités coopératives pour sa classe}

L'accessibilité d'un matériel pédagogique organisé dans une logique coopérative permet une meilleure appropriation (Louis, 1995). Cependant, il n'existe pas d'ouvrage spécifique pour le contexte genevois et les activités proposées nécessitent d'être adaptées aux objectifs du PER. Les enseignant-e-s doivent alors se repérer dans les différentes ressources et créer des tâches pertinentes pour leur contexte.

\section{Difficultés à gérer le temps de préparation et le temps en classe}

Le temps nécessaire apparaît comme un coût important des travaux de groupes coopératifs (Abrami et al., 2004; Buchs et al., 2017; Koutselini, 2008). Les enseignant-e-s rapportent que cette approche est chronophage lors de la préparation des activités hors temps d'enseignement (Blatchford et al., 2003) et sur temps d'enseignement (Gillies \& Boyle, 2010), et craignent par conséquent qu'elle ne permette pas de couvrir l'ensemble des contenus disciplinaires (Peyrat-Malaterre, 2011). 


\section{Les freins et Leviers perçus}

Le dernier objectif de cette étude est de questionner ces enseignant-e-s sur les leviers et les freins à la mise en place de la PC qu'ils perçoivent dans leur contexte. Abrami et al. (2004) suggèrent que la mise en place d'une innovation pédagogique dépend principalement de trois prédicteurs: la valeur accordée à cette innovation (Values), la réussite que les enseignant-e-s pensent atteindre dans leur contexte lors de la mise en œuvre (Expectancies) et le coût perçu lié à cette mise en œuvre. Rouiller et Lehraus (2008) quant à elles différencient des facteurs qu'elles classent comme dépendants de l'action enseignante et indépendants de l'action enseignante. Ces modèles soulignent la complexité que revêt l'appropriation de la PC.

\section{La perception des effets de la pédagogie coopérative}

Le modèle d'Abrami et al. (2004) met en évidence la nécessité de percevoir les bénéfices d'une innovation pédagogique pour sa mise en place. En effet, percevoir les retombées positives pour les élèves est une source de motivation pour les enseignant-e-s (Rouiller \& Howden, 2010, Antil et al., 1998).

\section{Adéquation avec les objectifs et les moyens d'enseignement}

Sharan (2010) met en évidence que les moyens d'enseignement officiels en vigueur se prêtent peu à des activités coopératives et proposent essentiellement des activités frontales ou individuelles. Des moyens d'enseignement basés sur la PC et adaptés aux objectifs d'apprentissage du contexte local pourraient réduire les coûts (Abrami et al., 2004) et favoriser la mise en ouvre (Buchs et al., 2017; Gillies 2008).

\section{Soutien}

Le soutien collégial, d'une part, apparait comme un levier important dans de nombreuses recherches (Abrami et al., 2004; Brody \& Davidson, 1998; Cooper \& Boyd, 1998; Gillies \& Boyle, 2010; Lotan, 2004; Rouiller \& Howden, 2010; Rouiller \& Lehraus, 2008; Sabourin \& Lehraus, 2008). Ce soutien prend la forme d'attitudes partagées ou d'échanges de pratiques et de ressources entre collègues, voire de formes de coopération entre enseignant-e-s.

D'autre part, la direction d'établissement peut faciliter les opportunités collaboratives en allouant du temps de réflexion entre enseignant-e-s et en soutenant explicitement la coopération comme une approche d'enseignement appropriée (Rolheiser \& Stevahn, 1998; Rouiller \& Howden, 2010). Les projets d'école et les plans d'action collectifs peuvent soutenir les objectifs coopératifs (Rolheiser \& Stevahn, 1998).

\section{Caractéristiques des élèves}

Les caractéristiques personnelles des élèves, leurs attitudes ou comportements peuvent influencer la mise en place de la PC de la part de l'enseignant-e qui 
craint que certains membres du groupe refusent de travailler à plusieurs (Abrami et al., 1994 ). De plus, il peut être plus complexe d'organiser des interactions simultanées avec des grandes classes. Le nombre d'élèves serait donc à considérer.

\section{Objectifs et questions de recherche}

L'objectif de l'étude est de documenter de quelle(s) manière(s) des enseignants qui ont manifesté un intérêt pour la PC en suivant une initiation se positionnent par rapport aux pratiques coopératives. Quatre questions de recherche sont étudiées:

1) Quel pourcentage de temps les enseignant-e-s déclarent-ils-elles dédier aux travaux de groupes coopératifs?

2) Dans quelle mesure déclarent-ils-elles suivre les principes de la PC lorsqu'ils mettent en place des travaux de groupes dits coopératifs?

3) Quelles sont les difficultés rapportées par ces enseignant-e-s?

4) Quels éléments perçoivent-ils-elles comme freinant ou facilitant la mise en œuvre dans leur contexte?

\section{Méthodologie}

\section{Participants et procédure}

Nous avons sollicité les enseignant-e-s primaires ayant suivi une initiation à la $\mathrm{PC}$ en formation continue entre 2006 et 2015. Cette formation se déroule sur une journée, suivie de deux demi-journées pour permettre l'expérimentation en classe par les enseignant-e-s. Il leur a été demandé de répondre à un questionnaire sur internet. Ce questionnaire a été envoyé à tous les enseignant-e-s (118) ayant participé à la formation continue. Les premiers questionnaires ont été envoyés en 2013 puis après les formations de 2014 et 2015. Les analyses ne montrant pas de différences, nous avons traité tous les questionnaires ensemble. Cette population a été choisie, car c'est elle qui est la plus à même d'utiliser cette approche et d'identifier les obstacles et leviers influençant la mise en pratique au-delà des connaissances de base. Soixante-six questionnaires ont été retournés ( $40.6 \%$ provenant d'enseignant-e-s de la division élémentaire et $59.4 \%$ de la division moyenne). Les effectifs pour chaque mesure peuvent varier compte tenu des non réponses à certaines questions.

\section{Mesures}

Le questionnaire a été construit en trois parties: pratiques coopératives rapportées, difficultés relevées, leviers et freins perçus dans leur contexte. Nous avons demandé aux enseignant-e-s de répondre pour l'année en cours. Les répondante-s exerçant dans plusieurs classes ont choisi la classe avec laquelle ils-elles se 
sentaient le plus confortables. Lorsque plusieurs questions renvoient au même construit, nous avons calculé l'indice de fidélité inter-items ( $\alpha$ de Cronbach). Lorsque cet indice est supérieur à .70, il indique des réponses suffisamment cohérentes pour pouvoir calculer un indice moyen.

\section{Pratiques coopératives rapportées}

\section{Pourcentage de temps dédié au travail de groupes coopératifs}

Les enseignant-e-s répondaient à la question suivante: "Sur l'ensemble du temps d'enseignement, estimez en pourcentage (\%) le temps durant lequel vous proposez du travail de groupe coopératif à vos élèves».

\section{L'importance accordée aux différents principes de la pédagogie coopérative}

Les enseignant-e-s devaient se positionner par rapport à l'affirmation suivante: «Lorsque je prépare une activité à réaliser en travail de groupe coopératif pour mes élèves, je veille à [ce que]...». Nous avons créé des questions pour chacun des principes de la PC présentés dans la partie théorique. Dans la mesure où l'interdépendance positive liée aux buts est indispensable et que les autres interdépendances dépendent des activités, nous nous sommes limités à cette première interdépendance. Les ancrages de l'échelle allaient de 1 Faux pour moi à 7 Vrai pour moi. Les questions et les indices de fidélité inter-items sont présentés dans la figure 1.

Pour les difficultés rapportées ainsi que les leviers et freins, les items ont été choisis au regard des études précédentes menées citées plus haut (difficulté à mettre en œuvre les différents principes, difficulté à modifier le lieu d'autorité, perception des effets de la pédagogie coopérative, etc.).

\section{Difficultés rapportées}

Les enseignant-e-s devaient se positionner par rapport au début d'affirmation: «Lorsque je propose du travail de groupe coopératif dans ma classe, je trouve qu'il est plutôt difficile/facile de...». Les ancrages de l'échelle allaient de 1 Difficile pour moi à 7 Facile pour moi. Les questions et les indices de fidélité inter-items sont présentés dans la figure 2.

\section{Leviers et freins perçus dans leur contexte}

Les enseignant-e-s se sont prononcés sur l'affirmation suivante: «Lorsque je propose du travail de groupe coopératif dans ma classe, je trouve que sa mise en place est plutôt freinée/facilitée par...». Les ancrages de l'échelle allaient de 1 Freine beaucoup ma mise en cuvre à 7 Facilite beaucoup ma mise en æuvre. Les questions et les indices de fidélité inter-items sont présentés dans la figure 3. 


\section{Figure 1. Questions permettant de documenter l'importance accordées aux diffé- rents principes de la pédagogie coopérative.}

\begin{tabular}{|c|c|}
\hline $\begin{array}{l}\text { Cette année, lorsque je prépare une activité à réaliser en travail de groupe } \\
\text { coopératif pour mes élèves, je veille à (ce que) }\end{array}$ & Principes \\
\hline $\begin{array}{l}\text {.. les membres d'un groupe s'encouragent mutuellement. } \\
\text {.. les élèves s'encouragent à s'exprimer lorsqu'ils travaillent. } \\
\ldots \text { les élèves soient incités à interagir ensemble. } \\
\ldots \text { faire travailler les élèves dans des groupes restreints (entre } 2 \text { et } 5 \text { membres). }\end{array}$ & $\begin{array}{l}\text { Interactions } \\
\text { constructives } \\
(\alpha=.77)\end{array}$ \\
\hline $\begin{array}{l}\ldots \text { instaurer un climat positif dans les groupes de travail avant qu'ils ne commencent } \\
\text { l'activité (activité favorisant la communication et le soutien entre les } \\
\text { participants). } \\
\ldots \text { travailler des comportements sociaux utiles dans les interactions avec les autres. } \\
\ldots \text { souder les membres des groupes ensemble (activité favorisant l'esprit d'équipe). } \\
\ldots \text { travailler explicitement certaines valeurs par exemple le respect, la confiance, } \\
\text { l'ouverture aux autres. }\end{array}$ & $\begin{array}{l}\text { Climat } \\
\text { constructif } \\
(\alpha=.87)\end{array}$ \\
\hline $\begin{array}{l}\text {... prendre du temps pour expliciter l'utilité d'une nouvelle habileté coopérative. } \\
\ldots \text { travailler une habileté coopérative à plusieurs reprises pour faciliter sa maîtrise. } \\
\ldots \text { construire un outil aide-mémoire permettant de mettre en œuvre en parole et en } \\
\text { comportements les différentes habiletés coopératives. } \\
\text {... travailler des habiletés coopératives utiles pour bien travailler ensemble. }\end{array}$ & $\begin{array}{l}\text { Habiletés } \\
\text { coopératives } \\
(\alpha=.85)\end{array}$ \\
\hline $\begin{array}{l}\text {.. les élèves réfléchissent sur les actions qui ont permis d'avancer durant l'activité. } \\
\ldots \text { les élèves réfléchissent sur ce qu'ils pourraient améliorer lors de leur prochaine } \\
\text { activité en groupe coopératif. } \\
\ldots \text { donner du temps aux élèves pour réfléchir à la manière dont a fonctionné leur } \\
\text { groupe. } \\
\text {... les élèves prennent du temps suite à l'activité pour réfléchir sur leur action dans le } \\
\text { groupe. }\end{array}$ & $\begin{array}{l}\text { Réflexion } \\
\text { Critique } \\
(\alpha=.84)\end{array}$ \\
\hline $\begin{array}{l}\text {... chaque membre d'un groupe perçoive qu'il ne peut atteindre son but que si tous } \\
\text { les membres de son groupe atteignent également le leur. } \\
\text {... les idées de chaque membre soient nécessaires pour bien réaliser la tâche de } \\
\text { groupe. } \\
\ldots \text { tous les membres de chaque groupe participent à la réalisation de l'activité } \\
\text { commune. }\end{array}$ & $\begin{array}{l}\text { Interdépendance } \\
\text { liée aux buts } \\
(\alpha=.73)\end{array}$ \\
\hline $\begin{array}{l}\text {... chacun s'assure que chaque membre de son groupe apprenne lors de l'activité. } \\
\ldots \text { chaque participant fasse de son mieux pour accomplir sa part de travail. } \\
\ldots \text { chaque membre contribue à la réalisation de l'activité. }\end{array}$ & $\begin{array}{l}\text { Responsabilisation } \\
\text { individuelle } \\
(\alpha=.76)\end{array}$ \\
\hline
\end{tabular}


Figure 2. Questions permettant de documenter les difficultés rapportées par les enseignants.

\begin{tabular}{|c|c|}
\hline $\begin{array}{l}\text { Cette année, lorsque je prépare une activité à réaliser en travail de groupe } \\
\text { coopératif pour mes élèves, je trouve qu'il est plutôt difficile/facile de... }\end{array}$ & Principes \\
\hline $\begin{array}{l}\ldots \text { travailler les habiletés coopératives nécessaires au travail de groupe coopératif. } \\
\ldots \text { faire réfléchir les élèves au fonctionnement des groupes. } \\
\ldots \text { enseigner aux élèves à coopérer. } \\
\ldots \text { créer les conditions pour que les élèves puissent coopérer. }\end{array}$ & $\begin{array}{l}\text { Préparer les élèves } \\
\text { à la coopération } \\
(\alpha=.84)\end{array}$ \\
\hline ... structurer la tâche pour rendre les élèves complémentaires. & Structurer \\
\hline $\begin{array}{l}\text {... faire clairement percevoir aux membres d'un groupe qu'ils sont } \\
\text { positivement liés les uns aux autres. }\end{array}$ & $\begin{array}{l}\text { l'interdépendance } \\
(\alpha=.76)\end{array}$ \\
\hline $\begin{array}{l}\text {. faire clairement percevoir aux membres d'un groupe qu'ils sont } \\
\text { responsables de leur travail. } \\
\ldots \text { structurer la tâche pour responsabiliser chaque membre. }\end{array}$ & $\begin{array}{l}\text { Structurer } \\
\text { responsabilité } \\
(\alpha=.75)\end{array}$ \\
\hline $\begin{array}{l}\text {... accepter de ne pas être au centre des interactions entre élèves. } \\
\ldots \text { accepter de ne pas savoir tout ce qui se construit dans les groupes. } \\
\ldots \text {.. laisser travailler les élèves en autonomie, sans ma supervision directe. } \\
\ldots \text { déléguer une part de l'enseignement aux élèves. } \\
\text {... faire confiance aux élèves. }\end{array}$ & $\begin{array}{l}\text { Gérer l'autorité } \\
(\alpha=.88)\end{array}$ \\
\hline $\begin{array}{l}\ldots \text { observer les élèves pendant le travail en groupes coopératifs. } \\
\ldots \text { réguler le fonctionnement des élèves dans des groupes coopératifs. } \\
\ldots \text { réguler les stratégies cognitives des élèves dans des groupes coopératifs. }\end{array}$ & $\begin{array}{l}\text { Réguler l'activité } \\
\text { des élèves } \\
(\alpha=91)\end{array}$ \\
\hline $\begin{array}{l}\text {.. répartir les élèves en groupes. } \\
\ldots \text { gérer simultanément l'avancement du travail des différents groupes. } \\
\ldots \text { gérer les différences de rythmes de travail des groupes. }\end{array}$ & $\begin{array}{l}\text { Gérer les groupes } \\
(\alpha=.73)\end{array}$ \\
\hline $\begin{array}{l}\text {.. évaluer les élèves de manière équitable. } \\
\ldots \text { évaluer les apprentissages de chaque élève à l'issue du travail de groupe. }\end{array}$ & $\begin{array}{l}\text { Evaluer } \\
(\alpha=83)\end{array}$ \\
\hline $\begin{array}{l}\text { dégager le temps nécessaire pour planifier des travaux de groupes } \\
\text { coopératifs. } \\
\ldots \text { dégager le temps nécessaire pour proposer du travail de groupe } \\
\text { coopératif aux élèves. }\end{array}$ & $\begin{array}{l}\text { Gérer le temps } \\
(\alpha=.92)\end{array}$ \\
\hline $\begin{array}{l}\text { me repérer dans les différentes ressources que j'ai à disposition lors de la } \\
\text { planification d'activités coopératives. } \\
\text {... trouver des pistes et/ou des idées d'activités coopératives pertinentes. }\end{array}$ & $\begin{array}{l}\text { Créer des activités } \\
\text { coopératives } \\
(\alpha=.85)\end{array}$ \\
\hline
\end{tabular}


Figure 3. Questions permettant de documenter les leviers et freins perçus par les enseignant-e-s dans leur contexte.

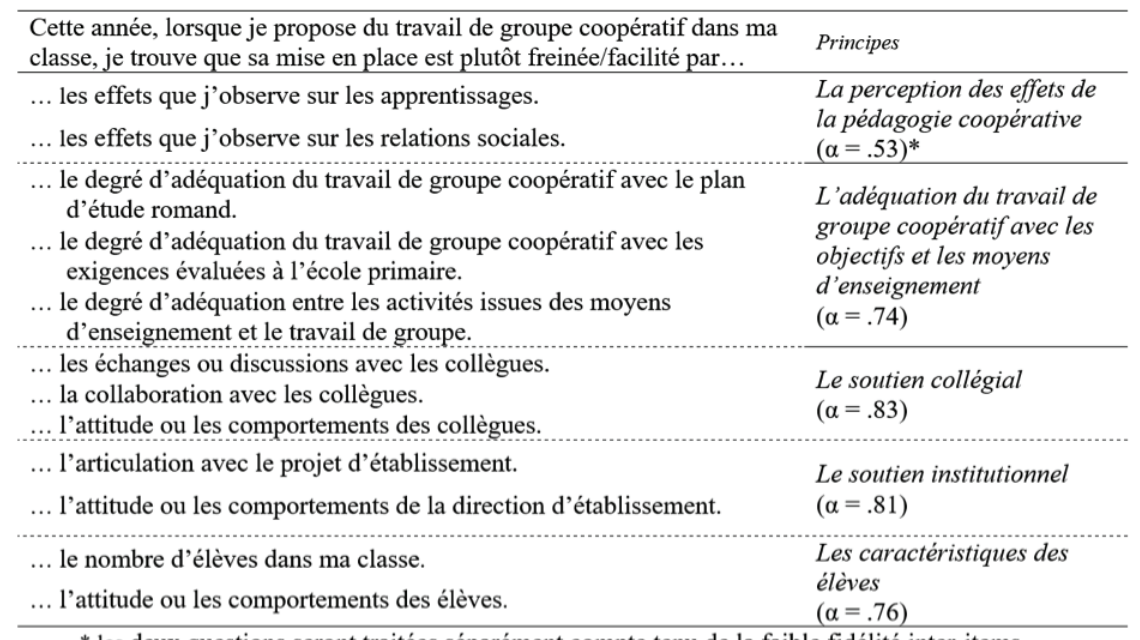

* les deux questions seront traitées séparément compte tenu de la faible fidélité inter-items

\section{Résultats et discussion}

\section{Pourcentage de temps alloué au travail de groupe coopératif}

Les enseignant-e-s rapportent entre $0 \%$ ( 2 personnes) et $75 \%$ (une personne) du temps d'enseignement dédié aux travaux de groupes coopératifs ( $M=23.46 \%$, SD $=15.64$; Médiane $=20)$. Ces enseignant-e-s disent ainsi passer en moyenne près d'un quart du temps d'enseignement avec des travaux coopératifs. Le tableau 1 présente la répartition des réponses.

Tableau 1. Fréquence des pourcentages de temps dédié aux travaux de groupes coopératifs.

\begin{tabular}{lcccccc}
\hline Réponse & $0 \%$ & $1-10 \%$ & $11-20 \%$ & $21-30 \%$ & $31-50 \%$ & $>50 \%$ \\
\hline Effectif & 2 & 17 & 17 & 15 & 12 & 2 \\
Pourcentage & 3.1 & 26.2 & 26.2 & 23.1 & 18.5 & 3.1 \\
\hline
\end{tabular}

Ce temps, relativement important, en le comparant aux $12 \%$ du temps dans les travaux de Baines et al. (2003) ou des 8\% observés par Pianta et al. (2007), peut s'expliquer par le fait que notre étude concerne des enseignant-e-s qui ont manifesté un intérêt pour la PC. Il se peut aussi que ce chiffre soit surévalué: les enseignant-e-s qui n'ont pas ou peu introduit la coopération après la formation 
peuvent ne pas avoir retourné le questionnaire. De plus, les enseignant-e-s déclarent eux-mêmes leurs pratiques.

\section{Importance accordée aux principes théoriques}

De manière générale, les moyennes pour la prise en compte des différents principes sont relativement élevées (voir le Tableau 2) avec un minimum de 4.83 pour la réflexion critique et un maximum de 5.55 pour la responsabilité individuelle sur une échelle de 1 à 7 . Les enseignant-e-s déclarent ainsi respecter les principes théoriques de la PC avec une moyenne au-dessus du milieu de l'échelle «4» pour tous les principes, Test $\mathrm{T}$ pour échantillon indépendant $>4.31$, ps < .001 .

Tableau 2. Statistiques descriptives concernant l'importance accordée (1: Faux pour moi - 7: Vrai pour moi) aux différents principes de la pédagogie coopérative (56 questionnaires complets).

\begin{tabular}{cccc} 
Moyenne & $\begin{array}{c}\text { Ecart- } \\
\text { type }\end{array}$ & $\begin{array}{c}\text { Min- } \\
\text { Max }\end{array}$ & $\begin{array}{c}\text { Test-T } \\
\text { échantillon } \\
\text { unique (4) }\end{array}$ \\
\hline
\end{tabular}

Interactions constructives

$\begin{array}{llll}5.42 & 1.23 & 2-7 & 8.60^{* * *}\end{array}$

Préparation des élèves pour la coopération

\begin{tabular}{lcccc} 
Climat constructif & 5.32 & 1.44 & $2-7$ & $6.89^{* * *}$ \\
Habiletés coopératives & 5.08 & 1.41 & $2-7$ & $5.75^{* * *}$ \\
Réflexion critique & 4.83 & 1.45 & $1-7$ & $4.31^{* * *}$ \\
Organisation des interactions & & & & \\
Interdépendance liée aux buts & 5.31 & 0.98 & $3-7$ & $10.03^{* * *}$ \\
Responsabilisation individuelle & 5.55 & 1.33 & $2-7$ & $8.74^{* * *}$ \\
\hline
\end{tabular}

Note: ${ }^{*} \mathrm{p}=.05,{ }^{* *} \mathrm{p}=.01,{ }^{* * *} \mathrm{p}=.001$ pour la différence significative avec le milieu de l'échelle (4) testée avec un t pour échantillon indépendant.

Ainsi, la formation semble avoir sensibilisé ces enseignant-e-s aux principes centraux de la PC. Les répondants rapportent faire particulièrement attention à ce qu'une responsabilité individuelle $(\mathrm{M}=5.55)$ et une interdépendance positive liée aux buts $(M=5.31)$ soient mises en place. S'ils prennent également en compte le climat $(M=5.32)$ et les interactions constructives $(M=5.42)$, ils sont moins attentifs aux principes liés aux habiletés coopératives $(M=5.08)$ et à la réflexion critique $(\mathrm{M}=4.83)$. Il se peut que les enseignant-e-s considèrent ces derniers éléments comme une étape supplémentaire dans un temps d'enseignement déjà trop court. La mise de côté de ces principes pourrait cependant réduire les bénéfices des activités coopératives (Topping et al., 2017). 
Le tableau 3 présente les corrélations entre le temps dédié aux travaux de groupe coopératifs et l'importance que les enseignant-e-s déclarent attribuer aux différents principes de la PC. Toutes les corrélations sont positives et significatives mais sont d'une ampleur modérée variant de .29 à .38. Ainsi, si le temps que les enseignant-e-s rapportent dédier aux travaux de groupe coopératifs est encourageant, ces corrélations soulignent que cette proportion de temps n'implique pas nécessairement qu'ils-elles accordent de l'importance à l'ensemble des principes préconisés par la PC. Comme l'indiquent les précédentes recherches, les enseignant-e-s s'écartent des propositions théoriques (Antil et al., 1998; Koutselini, 2008; Lehraus, 2002). Ce résultat pointe l'importance de ne pas se contenter du temps dédié aux travaux de groupe pour documenter les pratiques dans la mesure où les enseignant-e-s qui rapportent le plus de temps ne sont pas systématiquement les personnes qui accordent le plus d'importance aux principes théoriques.

Tableau 3. Corrélations entre le temps que les enseignant-e-s rapportent dédier aux travaux de groupes coopératifs et l'importance quils déclarent accorder aux principes de la pédagogie coopérative.

\begin{tabular}{|c|c|c|c|c|c|c|}
\hline & \multicolumn{3}{|c|}{$\begin{array}{l}\text { Préparation des élèves pour } \\
\text { la coopération }\end{array}$} & \multirow[b]{2}{*}{$\begin{array}{c}\text { Interactions } \\
\text { construc- } \\
\text { tives }\end{array}$} & \multicolumn{2}{|c|}{$\begin{array}{l}\text { Organisation des } \\
\text { interactions }\end{array}$} \\
\hline & Climat & $\begin{array}{l}\text { Habiletés } \\
\text { coopératives }\end{array}$ & $\begin{array}{l}\text { Réflexion } \\
\text { critique }\end{array}$ & & $\begin{array}{l}\text { Interdépen- } \\
\text { dance liée } \\
\text { aux buts }\end{array}$ & $\begin{array}{l}\text { Responsa- } \\
\text { bilisation }\end{array}$ \\
\hline $\begin{array}{l}\text { Temps dédié aux } \\
\text { travaux de groupe } \\
\text { coopératifs }\end{array}$ & $.29^{*}$ & $.29^{*}$ & $.31^{*}$ & $.38^{* * *}$ & $.38^{* * *}$ & $.33^{* *}$ \\
\hline
\end{tabular}

\section{Difficultés à mettre en œuvre les différents éléments} Le tableau 4 présente les statistiques descriptives concernant les difficultés rapportées par les enseignant-e-s, ainsi que le pourcentage de répondant-e-s considérant chaque élément comme plutôt difficile à intégrer dans les pratiques professionnelles (moyennes entre 1 et 3 ) ou plutôt faciles à intégrer dans les pratiques professionnelles (moyennes entre 5 et 7). Le tableau indique également si la moyenne des réponses pour chaque élément differe du milieu de l'échelle (4).

Lévaluation des élèves, le travail avec les groupes, la préparation des élèves à la coopération, la structuration de la responsabilisation et la création d'activités coopératives sont globalement perçus ni comme faciles ni comme difficiles. En revanche, les enseignant-e-s indiquent que le changement de lieu d'autorité et le rôle de régulateur sont plutôt faciles à mettre en œuvre. Les résultats soulignent que deux éléments restent difficiles à organiser: la structuration de l'interdépendance positive et la gestion du temps. 
Tableau 4. Statistiques descriptives pour les difficultés rapportées (1: difficile - 7: facile) par les enseignant-e-s lors de la mise en place des travaux de groupes coopératifs (52 questionnaires complets).

\begin{tabular}{lccccc}
\hline & Moyenne & $\begin{array}{c}\text { Ecart- } \\
\text { type }\end{array}$ & $\begin{array}{c}\text { Test-T } \\
\text { échantillon } \\
\text { unique }(4)\end{array}$ & $\begin{array}{c}\text { Plutôt } \\
\text { difficile } \\
(1-3)\end{array}$ & $\begin{array}{c}\text { Plutôt } \\
\text { facile } \\
(5-7)\end{array}$ \\
\hline Préparer les élèves à la coopération & 3.99 & 1.19 & -0.06 & $40.4 \%$ & $53.8 \%$ \\
Structurer l'interdépendance positive & 3.57 & 1.35 & $-2.32^{*}$ & $53.8 \%$ & $30.8 \%$ \\
Structurer de la responsabilité & 4.08 & 1.54 & 0.36 & $42.3 \%$ & $42.3 \%$ \\
Gérer l'autorité & 4.81 & 1.39 & $4.21^{* * *}$ & $26.9 \%$ & $69.2 \%$ \\
Réguler l'activité des élèves & 4.41 & 1.34 & $2.20^{*}$ & $32.7 \%$ & $59.6 \%$ \\
Gérer les groupes & 4.16 & 1.19 & 0.97 & $42.3 \%$ & $44.2 \%$ \\
Evaluer les élèves & 3.85 & 1.54 & -0.72 & $42.3 \%$ & $38.5 \%$ \\
Créer des activités coopératives & 3.72 & 1.41 & -1.43 & $51.9 \%$ & $32.7 \%$ \\
Gérer le temps & 3.11 & 1.55 & $-4.16^{* * *}$ & $61.5 \%$ & $21.2 \%$ \\
\hline
\end{tabular}

Note: ${ }^{*} \mathrm{p}=.05,{ }^{* *} \mathrm{p}=.01,{ }^{* * *} \mathrm{p}=.001$ pour la différence significative avec le milieu de l'échelle (4) testée avec un t pour échantillon indépendant

Le tableau 5 présente les corrélations entre les difficultés potentielles et le pourcentage de temps que les enseignant-e-s déclarent dédier aux travaux de groupe coopératifs. Les résultats soulignent que les enseignant-e-s qui rapportent dédier le plus de temps aux travaux de groupes coopératifs sont également ceuxcelles qui trouvent le plus facile de préparer les élèves à la coopération, de dégager le temps nécessaire à la mise en œuvre des travaux de groupes coopératifs et de créer des activités coopératives.

Tableau 5. Corrélations entre les difficultés potentielles et le pourcentage de temps que les enseignant-e-s déclarent dédier aux travaux de groupes coopératifs.

\begin{tabular}{|c|c|c|c|c|c|c|c|c|c|}
\hline & $\begin{array}{l}\text { Prépa- } \\
\text { ration } \\
\text { coopé- } \\
\text { ration }\end{array}$ & $\begin{array}{l}\text { Interdé- } \\
\text { pendance } \\
\text { positive }\end{array}$ & $\begin{array}{l}\text { Respon- } \\
\text { sabili- } \\
\text { sation }\end{array}$ & $\begin{array}{l}\text { Auto- } \\
\text { rité }\end{array}$ & $\begin{array}{l}\text { Régu- } \\
\text { lation }\end{array}$ & $\begin{array}{l}\text { Grou- } \\
\text { pes }\end{array}$ & $\begin{array}{l}\text { Evalu- } \\
\text { ation }\end{array}$ & $\begin{array}{l}\text { Acti- } \\
\text { vités }\end{array}$ & Temps \\
\hline $\begin{array}{l}\text { Temps dédié aux } \\
\text { travaux de groupe } \\
\text { coopératifs }\end{array}$ & $.39^{* *}$ & $.24^{\dagger}$ & $.26^{\dagger}$ & .14 & $.27^{\dagger}$ & -.02 & .06 & $.47^{* *}$ & $.42^{* *}$ \\
\hline \multicolumn{10}{|c|}{ Note: ${ }^{\dagger} \mathrm{p}<.10,{ }^{*} \mathrm{p}=.05,{ }^{* *} \mathrm{p}=.01$} \\
\hline \multicolumn{10}{|c|}{$\begin{array}{l}\text { Ces résultats soulignent le défi que représente la gestion du temps dans l'ensei- } \\
\text { gnement. Non seulement les enseignant-e-s trouvent difficile de dégager le } \\
\text { temps nécessaire, mais cette difficulté prédit le temps consacré aux travaux de } \\
\text { groupes coopératifs. En effet, avec une organisation magistrale d'une activité, } \\
\text { l'enseignant est le principal détenteur de l'avancée didactique du travail. Cette } \\
\text { hypothèse est soutenue par le fait que les enseignant-e-s qui éprouvent le moins } \\
\text { de difficulté à gérer le temps rapportent consacrer plus de temps aux travaux de } \\
\text { groupes coopératifs. }\end{array}$} \\
\hline
\end{tabular}


Leviers et freins perçus pour la mise en œuvre de la pédagogie coopérative dans leur contexte

Les enseignant-e-s mettent en avant un seul frein pour la mise en place de la PC dans leur contexte quotidien (Tableau 6), à savoir l'adéquation du travail de groupe coopératif avec les objectifs d'apprentissage et moyens d'enseignement. Malgré les recommandations institutionnelles (CIIP, 2010), les moyens d'enseignement actuellement en vigueur proposent le plus souvent des activités individuelles (fiche, exercice d'un livre, etc.). Cela demande aux enseignant-e-s du temps supplémentaire pour créer des activités coopératives en lien avec les objectifs d'apprentissage.

Les caractéristiques des élèves apparaissent relativement neutres (la moyenne ne differe pas du milieu de l'échelle). Il est intéressant de noter que les enseignant-e-s perçoivent un certain nombre de facteurs qui facilitent la mise en œuvre de la PC (dans l'ordre croisant d'importance): la perception des effets sur les apprentissages et les relations sociales, la collaboration avec les collègues et le contexte de l'établissement scolaire.

\section{Tableau 6. Statistiques descriptives pour les freins et leviers perçus dans les} pratiques quotidiennes (1: Freine beaucoup- 7: Facilite beaucoup ma pratique, 52 questionnaires complets).

\begin{tabular}{|c|c|c|c|c|c|}
\hline & Moyenne & Ecart-type & $\begin{array}{c}\text { Test-T } \\
\text { échantillon } \\
\text { unique (4) }\end{array}$ & $\begin{array}{l}\text { Plutôt frein } \\
\quad(1-3)\end{array}$ & $\begin{array}{l}\text { Plutôt levier } \\
\quad(5-7)\end{array}$ \\
\hline Caractéristiques des élèves & 3.87 & 1.60 & -0.61 & $42.3 \%$ & $38.5 \%$ \\
\hline $\begin{array}{l}\text { Perception des effets sur les } \\
\text { apprentissages }\end{array}$ & 4.44 & 1.39 & $2.29^{*}$ & $26.9 \%$ & $51.9 \%$ \\
\hline $\begin{array}{l}\text { Perception des effets sur les } \\
\text { relations sociales }\end{array}$ & 4.98 & 1.26 & $5.61^{* * *}$ & $15.4 \%$ & $69.2 \%$ \\
\hline $\begin{array}{l}\text { Adéquation du travail de } \\
\text { groupe coopératif avec objectifs } \\
\text { et les moyens d'enseignement }\end{array}$ & 3.68 & 1.17 & $-1.98^{*}$ & $55.8 \%$ & $28.8 \%$ \\
\hline Soutien collégial & 4.88 & 1.29 & $4.95^{* * *}$ & $13.5 \%$ & $67.3 \%$ \\
\hline Soutien institutionnel & $5.05^{*}$ & 1.47 & $5.13^{* * *}$ & $15.4 \%$ & $65.4 \%$ \\
\hline
\end{tabular}

Ainsi, conformément aux propositions d'Abrami et al. (2004), les effets perçus sont des éléments favorisant son appropriation. Nos résultats confirment également l'importance du soutien que ce soit de la part des pairs (Brody \& Davidson, 1998; Cooper \& Boyd, 1998; Gillies \& Boyd, 2010; Lotan, 2004) ou de la direction (Rolheiser \& Stevahn, 1998; Rouiller \& Howden, 2010). En effet, les orientations données par les directions d'établissements et leurs attitudes jouent un grand rôle dans l'appropriation d'une nouvelle approche pédagogique et favorisent la prise de risque. Selon Louis (1995), la direction possède un rôle important, car elle peut soutenir l'enseignant-e en s'informant 
de l'état de sa démarche et en lui demandant de la présenter aux collègues. Puis, le fait d'échanger et de réfléchir avec des pairs est perçu comme favorisant une meilleure mise en place de l'approche coopérative.

\section{Conclusion}

Le questionnaire créé dans la présente enquête contribue à l'étude de la mise en œuvre de la PC en fournissant un outil pour mesurer l'importance accordée par les enseignant-e-s aux différents principes, les difficultés qu'ils perçoivent ainsi que les freins et leviers qu'ils identifient dans leur contexte. Nos résultats soulignent que les répondant-e-s déclarent veiller à favoriser les principes communs de l'apprentissage coopératif, à savoir les interactions constructives, l'interdépendance liée aux buts et la responsabilisation. Ce constat encourageant est cependant nuancé par deux autres résultats. Premièrement, si les moyennes sont supérieures au milieu de l'échelle, elles restent modérées. Deuxièmement, la plus forte corrélation entre le temps dédié aux travaux coopératifs et l'importance accordée aux principes n'excède pas .38, ce qui est cohérent avec les relations reportées par Abrami et al. (2004), mais dénote que les principes sont appropriés de manière variable lors des travaux de groupes.

\section{Limites de L'étude}

Il convient de souligner les principales limites de cette étude. Tout d'abord, le nombre de répondant-e-s est relativement faible, ce qui invite à la prudence dans la généralisation des résultats. Ensuite, l'étude a porté sur les pratiques déclarées des enseignant-e-s, ce qui peut différer des pratiques réelles. En effet, des écarts peuvent apparaître entre ce que disent et font les enseignant-e-s. De plus, il se peut que ceux-ci aient oublié certains savoirs sur le sujet ou n'aient pas eu suffisamment de temps pour se les approprier. Cependant, l'objectif de cette étude est de documenter les pratiques coopératives chez des enseignant-e-s qui, à un moment de leur carrière, se sont intéressés à la pédagogie coopérative.

\section{Des voies pour favoriser l'appropriation de la pédagogie coopérative}

Même si la validité des résultats de cette présente étude peut être discutée au regard des limites présentées précédemment, il est toutefois possible de dégager certaines conclusions. Les résultats soulignent que la structuration de l'interdépendance positive et la gestion du temps représentent toujours des points complexes après une initiation. La structuration de l'interdépendance positive étant centrale pour la qualité des interactions, il est important d'acquérir des stratégies permettant de renforcer l'efficacité et le sentiment de compétence des enseignant-e-s quant à cette dimension (Abrami et al., 2004). De plus, les enseignant-e-s qui indiquent davantage qu'il est facile de créer des activités coopéra- 
tives et de gérer le temps sont également les personnes qui rapportent consacrer le plus de temps aux travaux de groupes coopératifs.

Les effets perçus de la PC, tout comme le soutien collégial ou institutionnel, sont considérés comme des éléments qui facilitent l'appropriation de la PC. À contrario, l'adéquation des travaux de groupes avec le cadre institutionnel que représentent la progression des apprentissages déterminés par le PER ou les moyens d'enseignement sont perçus comme peu propices à la mise en œuvre de la PC. L'ensemble de ces éléments permet d'esquisser quelques pistes pour la formation professionnelle en termes d'organisation et de contenus.

\section{Des voies pour la formation des enseignant-e-s}

L'organisation de la formation continue que ces enseignant-e-s ont suivie pourrait être améliorée. L'initiation de deux jours offre un programme combinant des apports théoriques et des expériences coopératives avec des exemples et des moments de réflexion. L'objectif est d'apporter les connaissances des principes de la PC, de présenter les effets de cette pédagogie sur la qualité des relations sociales et des apprentissages scolaires. Ainsi, pour renforcer cette prise de conscience, les participant-e-s sont amené-e-s à expérimenter des activités en séance et à introduire des activités dans leur classe.

Cependant, son organisation ne semble pas optimale si on se réfere aux écrits de Brody et Davidson (1998) et Lotan (2004). En effet, les enseignant-e-s s'inscrivent de manière individuelle à cette formation et, de retour dans leur école, le soutien de l'équipe et de la direction peut s'avérer limité freinant ainsi la mise en œuvre. Nos résultats relatifs à l'importance du soutien collégial et institutionnel militent pour des formations d'établissement où tous les enseignant-e-s de l'école sont formé-e-s ensemble. Ce type de formation a l'avantage de favoriser les échanges entre enseignant-e-s qui partagent des connaissances et une culture communes ainsi que des orientations soutenues par la direction de l'établissement. Dans ces conditions, le temps de formation permettrait d'intégrer des planifications communes entre collègues (Brody \& Davidson, 1998) et favoriserait une planification moins coûteuse en temps, la préparation des différentes activités coopératives étant répartie sur les enseignant-e-s exerçant dans le même degré. Une telle organisation initiée lors de la formation pourrait perdurer dans l'école et favoriser un engagement à long terme.

De plus, ces formations pourraient intégrer un accompagnement en classe par le/la formateur-trice et par les collègues (Cohen, 2002) et favoriser l'échange de pratiques et de ressources (Rouiller \& Howden, 2010). Le fait de pouvoir partager les difficultés et trouver un soutien auprès de ses collègues permet de progressivement inclure la coopération dans les pratiques courantes et d'encourager les enseignant-e-s à prendre des risques. Certain-e-s chercheur-euse-s tendent à affirmer qu'un enseignant-e possédant un statut d'expert quant à la PC permettrait un soutien plus efficace (Brody, 2015). 
En termes de contenus, ces formations devraient permettre aux enseignant-e$s$ de travailler la manière de structurer l'interdépendance positive dans des tâches issues des moyens d'enseignement officiels. Il est ainsi important de disposer de manuels, de ressources et de matériel adaptés aux objectifs d'apprentissage du PER (Hattie, 2008; Johnson \& Johnson, 2009; Kyndt et al., 2013; Slavin, 2014). Les planifications mutualisées lors de la formation favorisent la mise en ouvre immédiate et permet de gagner ce temps si précieux. Elles permettent également de fournir une banque d'activités préalablement pensées selon les principes de la PC et organisée en fonction d'une typologie des tâches scolaires demandées aux élèves. Cette banque pourrait être initiée lors de la formation continue, enrichie au sein de l'école et testée par les enseignant-e-s pour être ensuite mise à disposition d'une communauté enseignante plus large permettant à un maximum d'enseignant-e-s de pouvoir organiser leur enseignement en intégrant régulièrement des activités coopératives sans que le temps de préparation soit un frein.

\section{Références}

Abrami, P. C., Chambers, B., Poulsen, C., Kouros, C., Farrell, M., \& D’Apollonia, S. (1994). Positive social interdependence and classroom climate. Genetic, Social, and General Psychology Monographs, 120(3), 329-346.

Abrami, P. C., Poulsen, C. \& Chambers, B. (2004). Teacher motivation to implement an educational innovation: factors differentiating users and non-users of cooperative learning. Educational psychologist, 24(2), 201-216.

Abrami, P.C., Chambers, B., Poulsen, C., De Simone, C., d'Apollonia, S., \& Howden, J. (1996). L'apprentissage coopératif: Théories, méthodes, activités (trad). Montréal: Les Editions de la Chenelière.

Antil, L. R., Jenkins, J. R., Wayne, S. K. \& Vadasy, P. F. (1998). Cooperative learning: Prevalence, conceptualizations, and the relation between research and practice. American Educational Research Journal, 3(35), 419-454.

Baines, E., Blatchford, P. \& Kutnick, P. (2003). Changes in grouping practices over primary and secondary school. International Journal of Educational Research, 39, 9-34.

Baines, E., Rubie-Davies, C. \& Blatchford, P. (2009). Improving pupil group work interaction and dialogue in primary classrooms: Results from a year-long intervention study. Cambridge Journal of Education, 39(1), 95-117.

Blatchford, P., Kutnick, P., Baines, E. \& Galton, M. (2003). Toward a social pedagogy of classroom group work. International Journal of Educational Research, 39(1-2), 153-172.

Brody, C. (1998). The significance of teacher beliefs for professional development and cooperative learning. In M. Brody \& N. Davidson (Éds.), Professional development for cooperative learning. Issues and approaches (25-48). Albany: State University of New York.

Brody, C. (2015, octobre). Cooperative Learning for Social-Emotional Competence. Communication présentée à la Conférence IASCE, Cooperative Learning: Meeting the challenges of the $21^{\text {st }}$ century, Odense, Danemark.

Buchs, C. \& Butera, F. (2015). Cooperative learning and social skills development. In R. Gillies (Éd.), Collaborative learning: Developments in research and practice (pp. 201-217). New York Nova Science.

Buchs, C., Filippou, D., Pulfey, C. \& Volpé, Y. (2017). Challenges for cooperative learning implementation: reports from elementary school teachers. Journal of Education for Teaching, 43 (3), 296-306. 
Buchs, C., Lehraus, K. \& Crahay, M. (2012). Coopération \& apprentissage. In M. Crahay (Éd.), Peut-on lutter contre l'échec scolaire? (pp. 416-488). Bruxelles: De Boeck.

Brody, C. \& Davidson, N. (Éds.) (1998). Professional development for cooperative leaning: Issues and approaches. Albany: Sunny Press.

Cohen, E.G. (1994/1994). Le travail de groupe. Stratégies d'enseignement pour la classe hétérogène (trad. par F. Ouellet). Montréal: La Chenelière McGraw Hill.

Cohen, E. G. (2002). La construction sociale de l'équité dans les classes. In F. Ouellet (Éd.), Les défis du pluralisme en éducation (pp. 141-162). Laval: Les presses de l'université Laval.

Conférence intercantonale de l'instruction publique (2010). Description des capacités transversales. In Plan d'études romand. Neuchâtel: CIIP. Repéré à https://www.plandetudes.ch/ web/guest/capacites-transversales $1 \#$ coll

Connac, S. (2010). Apprendre avec les pédagogies coopératives. Démarches et outils pour l'école. Paris: ESF Editeur.

Cooper, C. \& Boyd, J. (1998). Creating sustained professional growth through collaborative reflection, In M. Brody \& N. Davidson (Éds.), Professional development for cooperative learning. Issues and approaches (pp. 49-62). Albany: State University of New York Press.

Davidson, N., \& Major, C. H. (2014). Boundary crossings: Cooperative learning, collaborative learning, and problem-based learning. Journal on Excellence in College Teaching, 25(3 $\& 4), 7-55$.

Foray, P. (2016). Devenir autonome. Apprendre à se diriger soi-même. Paris: ESF.

Gillies, R. M. \& Boyle, M. (2010). Teachers' reflections on cooperative learning: Issues of implementation. Teaching and teacher Education, 26, 933-940.

Hattie, J. (2008). Visible learning: A synthesis of over 800 meta-analyses relating to achievement. New York: Routledge.

Johnson, D. W. \& Johnson, R. T. (2009). An educational psychology success story: social interdependence theory and cooperative learning. Educational Researcher, 38(5), 365-379.

Johnson, D. W., Johnson, R. T., \& Holubec, E. J. (2008). Cooperation in the classroom (8e Éd.). Edina, MN: Interaction Book Company.

Kagan, S. (2013). Kagan cooperative learning structures. San Clemente: Kagan Publishing.

Koutselini, M. (2008). Teacher misconceptions and understanding of cooperative learning: an intervention study. Journal of Classroom Interaction, 43(2), 34-44.

Kutnick, P., Ota, C. \& Berdondini, L. (2008). Improving the effects of group working in classrooms with young school-aged children: facilitating attainment, interaction and classroom activity. Learning and Instruction, 18(1), 83-95.

Kyndt, E., Raes, E., Lismont, B., Timmers, F., Cascallar, E. \& Dochy, F. (2013). A metaanalysis of the effects of a face-to-face cooperative learning. Do recent studies falsify or verify earlier findings? Educational Research Review, 10, 133-149.

Lehraus, K. (2002). La pédagogie coopérative: de la formation à la mise en pratique. Revue Suisse des sciences de l'éducation, 24(3), 1-22.

Leutenegger, F. (2012). Un point de vue didactique sur le soutien pédagogique. Éducateur, $1,5-7$.

Lotan, R.A. (2004). Stepping into groupwork. In E.G. Cohen, C.M. Brody \& M. SaponShevin (Éds.), Teaching cooperative learning. The challenge for teacher education (pp. 167-182). Albany: State University of New York.

Louis, R. (1995). Les facteurs qui influent sur l'adoption par les enseignants d'une nouvelle approche pédagogique: le cas de la pédagogie coopérative. Vie pédagogique, 96, 47-50.

Peyrat-Malaterre, M.-F. (2011). Comment faire travailler efficacement des élèves en groupe? Tutorat et apprentissage coopératif. Bruxelles: De Boeck.

Pianta, R. C., Belsky, J., Houts, R. \& Morrison, F. (2007). Opportunities to learn in America’s elementary classrooms. Science, 315, 1795-1796. 
Rolheiser C. \& Stevahn, L. (1998). The role of staff developers in promoting effective teacher decision.making. In M. Bordy \& N. Davidson (Éds.), Professional development for cooperative learning. Issues and approaches (pp. 63-78). Albany: State University of New York.

Rouiller, Y. \& Howden, J. (2010). La pédagogie coopérative. Reflets de pratiques et approfondissements. Montréal: Chenelière McGraw Hill.

Rouiller, Y. \& Lehraus, K. (2008). Conclusion: Vers une modélisation des conditions favorables aux apprentissages coopératifs. In Y. Rouiller \& K. Lehraus (Éds.), Vers des apprentissages en coopération: rencontres et perspectives (pp. 221-237). Berne: Peter Lang.

Sabourin, M. \& Lehraus, K. (2008). Former des enseignants primaires à une approche coopérative: bilans et perspectives. In Y. Rouiller \& K. Lehraus (Éds.), Vers des apprentissages en coopération: rencontres et perspectives (pp. 191-220). Berne: Peter Lang.

Sharan, S. (Ed.) (1999). Handbook of cooperative learning methods. Westport: Greenwood publishing group.

Sharan, Y. (2010). Cooperative learning for academic and social gains: values pedagogy problematic practice. European Journal of Education, 2(45), 300-310.

Slavin, R. E. (1990). Cooperative learning: theory, research, and practice. Boston: Allyn \& Bacon.

Toczek, M.-C. (2004). Optimiser le travail en groupe: Le groupe classe - le groupe d'apprentissage. In M.-C. Toczek-Capelle \& D. Martinot (Eds.), Le défi éducatif. Des situations pour réussir (pp. 117-139). Paris: Armand Colin.

Topping, K., Buchs, C., Duran, D. \& van Keer, H. (2017). Effective peer learning. From principles to practical implementation. New York: Routledge.

Mots-clés: Pédagogie coopérative, apprentissage coopératif, formation continue, pratiques professionnelles, changement de pratiques

\section{Kooperatives Lernen: deklarierte Praxis und Aneignung Faktoren}

\section{Zusammenfassung}

Diese Forschungsarbeit dokumentiert den Einsatz kooperativer Lernmethoden bei Lehrer/innen, die eine Weiterbildung absolviert haben. Diese Lehrer/innen berichten, im Durchschnitt 25\% ihrer Unterrichtszeit für kooperative Gruppenarbeiten aufzuwenden. Sie geben an, dass sie die Schüler/innen anleiten zu kooperieren und ihre Interaktionen gemäss den Prinzipen dieser Methode organisieren. Allerdings, ist die Übereinstimmung zwischen den genannten Variablen gering. Die Befragten geben auch an, dass sie Schwierigkeit haben, die benötigte Zeit einzuschätzen und positive Interdependenz zu operationalisieren. Sie sehen Lernziele und Lehrmittel eher als Hindernisse für die Praxis an; die Wahrnehmung des Nutzens dieser Methode für die Schüler/innen und kollegiale und institutionelle Unterstützung betrachten sie als wesentliche Antriebskraft.

Schlagworte: Kooperatives Lernen, Weiterbildung, professionelle Praktiken, Änderung der Praktiken 


\section{Pedagogia cooperativa: pratiche dichiarate e fattori d'appropriazione}

\section{Riassunto}

Questa ricerca documenta l'appropriazione della pedagogia cooperativa da parte degli-delle insegnanti che hanno seguito dei corsi di formazione di iniziazione a questa pedagogia durante la formazione continua. Questi-e insegnanti affermano istituire dei lavori di gruppo cooperativi durante in media $25 \%$ del tempo d'insegnamento e dichiarano preparare gli-le allievi-e a cooperare e a organizzare le loro interazioni secondo i principi di questa pedagogia. Ciò malgrado il legame tra le variabili precedenti è debole. Le persone che hanno risposto alle nostre domande esprimono difficoltà a gestire il fattore tempo e a rendere operativa l'interdipendenza positiva. Identificano gli obbiettivi d'apprendimento e i mezzi d'insegnamento come dei freni alle loro pratiche pedagogiche; la percezione dei benefici per gli allievi così come il sostegno collegiale e istituzionale sono considerati come delle leve.

Parole-chiave: Pedagogia cooperativa, apprendimento cooperativo, formazione continua, prassi professionali, cambiamenti di prassi pedagogica

\section{Cooperative learning: reported practices and appropriation factors}

\section{Summary}

This study documents the appropriation of cooperative learning by teachers who followed an initiation in continuous training. The teachers report an average of $25 \%$ of their teaching time setting up cooperative group work. They declare preparing pupils to cooperate and organizing their interactions under this pedagogy's principles. However, the link between these previous variables is weak. Respondents express difficulties to manage time and operationalize positive interdependence. They identify learning goals and teaching methods as a weigh on their practice; the perception of benefits for pupils as well as collegial and institutional support are considered as leverages.

Keywords: Cooperative learning, continuous training, professional practices, change of practices 
Yann Volpé, enseignant primaire et chargé d'enseignement suppléant en sciences de l'éducation à l'Université de Genève.

Domaines de recherche : pédagogie coopérative, développement professionnel, identité enseignante

Université de Genève, Faculté de psychologie et des sciences de l'éducation FPSE, Boulevard du Pont-d'Arve 40, CH-1204 Genève

E-Mail: Yann.Volpe@unige.ch

Céline Buchs, docteure en psychologie sociale et maître d'enseignement et de recherche en sciences de l'éducation à l'Université de Genève. Domaines de recherche : Processus sociocognitifs et interactions sociales, les recherches portent principalement sur la régulation sociale des apprentissages et la pédagogie coopérative. Cette recherche a été soutenu par le fond national de la recherche avec le subside CRSII1_14172.

Université de Genève, Faculté de psychologie et des sciences de l'éducation FPSE, Boulevard du Pont-d'Arve 40, CH-1204 Genève

E-Mail: Celine.Buchs@unige.ch 